\title{
Initial value constraints with tensor matter
}

\author{
Ted Jacobson \\ Maryland Center for Fundamental Physics \\ Department of Physics, University of Maryland \\ College Park, MD 20742-4111, USA
}

\begin{abstract}
In generally covariant metric gravity theories with tensor matter fields, the initial value constraint equations, unlike in general relativity, are in general not just the $0 \mu$ components of the metric field equation. This happens because higher derivatives can occur in the matter stress tensor. A universal form for these constraints is derived here from a generalized Bianchi identity that includes matter fields. As an application, the constraints for Einstein-aether theory are found.
\end{abstract}

\section{Introduction}

A field theory with diffeomorphism symmetry can not have deterministic field equations. Although Einstein briefly thought this meant that a physical theory could not be diffeomorphism invariant, he soon realized that the observables may be deterministically evolved, even though the fields are not [1]). The solutions must involve four free functions (in four spacetime dimensions), which correspond to the freedom to apply an arbitrary time dependent diffeomorphism to the fields. This means that out of the collection of Euler-Lagrange equations obtained from varying the fields in the action, there must be four combinations that are not independent of the others. These are initial value constraint equations, and the remaining field equations imply that if the constraints hold initially, they are automatically preserved in time.

This is all very familiar to anyone who has worked with general relativity, but there is a twist if the matter energy-momentum tensor involves second time derivatives of the "matter" fields, as happens if the matter action involves covariant derivatives of tensor fields. In that case, the initial value constraints are not just the $0 \mu$ components of the metric field equations; instead, one must add to those a certain combination of the matter field equations. An example is Einstein-aether theory, in which the metric is coupled to a unit timelike vector field. 
In this article the form of the constraint equations in the presence of tensor matter is derived from a generalization of the Bianchi identity that includes the matter fields. It is then applied to three examples that illustrate different features. In particular, the constraints for Einstein-aether theory are obtained. After writing this up I learned that the result is (of course) not new. A paper by Bergmann 2 from 1949 obtains the same result, and it probably goes back even further. It was recently derived by Seifert and Wald[3], and applied to Einstein-aether theory in [4. However, it appears not to be common knowledge today. Since theories with tensor matter are currently of interest, it seems worth publishing this brief note, which aims to explain the result and make it easily accessible using low brow, coordinate based methods.

\section{General relativity}

To begin with let us review how it works in general relativity.

\subsection{Vacuum}

The vacuum Einstein equation,

$$
G^{\alpha \beta}=0,
$$

involves 10 independent components, matching the 10 independent components of the spacetime metric. However 4 of the 10 equations packaged into (11) must not actually be evolution equations. In fact, for any coordinate system $x^{\alpha}=\left(x^{0}, x^{i}\right)(i=1,2,3)$, the $0 \beta$ components of (1) involve no second derivatives with respect to $x^{0}$. (I will refer to $x^{0}$ as the "time" in the following, although in fact it can be any coordinate.) A simple way to see this 1 is from the Bianchi identity,

$$
\nabla_{\alpha} G^{\alpha \beta}=0
$$

which in component form reads

$$
\partial_{0} G^{0 \beta}+\partial_{i} G^{i \beta}+\Gamma_{\alpha \sigma}^{\alpha} G^{\sigma \beta}+\Gamma_{\alpha \sigma}^{\beta} G^{\alpha \sigma}=0 .
$$

\footnotetext{
${ }^{1}$ The argument is given this way in Weinberg's 1972 book $[5]$. It also appears in the 1975 english edition of Landau and Lifshitz's vol. 2 [6], which is translated from the 1973 russian 6th edition, but not in the 1971 english edition. For a chronicle of the early history see Stachel[7].
} 
If $G^{0 \beta}$ were to have second time derivatives, then the first term in (3) would have third time derivatives, unlike any other term. This would be inconsistent with (3) being true as an identity. Therefore we can infer that $G^{0 \beta}$ has no second time derivatives. The equations

$$
G^{0 \beta}=0
$$

are therefore not evolution equations but rather constraints on initial data. Moreover, the Bianchi identity is a first order differential equation $\partial_{0} G^{0 \beta}=$ $\ldots$, which implies that if these constraints hold at some initial time, and the rest of the field equations $G^{i j}=0$ hold at all times, then the constraints hold for all times.

It is no accident that the Bianchi identity is so intimately tied to the existence and preservation of the constraint equations. That identity can be viewed as a direct consequence of the diffeomorphism invariance of the Einstein-Hilbert action, $S=\int R$. To reduce clutter, the metric volume element $\sqrt{-g} d^{4} x$ is implicit here and in the following. (When the metric is varied, $\sqrt{-g}$ must certainly be varied as well.) Under a metric variation $\delta g_{\alpha \beta}$ the action varies as 2

$$
\delta S=-\int G^{\alpha \beta} \delta g_{\alpha \beta}
$$

If the metric variation arises from an infinitesimal diffeomorphism generated by a vector field $\xi^{\beta}$ we have

$$
\delta_{\xi} g_{\alpha \beta}=\mathcal{L}_{\xi} g_{\alpha \beta}=2 \nabla_{(\alpha} \xi_{\beta)}
$$

where $\mathcal{L}$ denotes the Lie derivative, and the round brackets on indices denote symmetrization. Substituting (6) in (5), integrating by parts, and assuming $\xi^{\beta}$ vanishes at the boundaries, we find that a diffeomorphism generates the variation

$$
\delta_{\xi} S=\int\left(2 \nabla_{\alpha} G^{\alpha \beta}\right) \xi_{\beta}
$$

Since the Einstein-Hilbert action is a spacetime scalar, $\delta_{\xi} S$ must vanish for all $\xi^{\beta}$, which implies the Bianchi identity (2). That is, the action is automatically stationary under those variations of the metric that arise from diffeomorphisms, so the corresponding Euler-Lagrange equations do not impose any conditions. The identity implied by this symmetry indicates which four of the field equations are constraints rather than evolution equations, and ensures that those constraints are preserved in time.

\footnotetext{
${ }^{2}$ We use the standard notation: indices are lowered and raised with the metric and inverse metric, $R=g^{\alpha \beta} R_{\alpha \beta}$ is the Ricci scalar, $R_{\alpha \beta}=R_{\alpha \sigma \beta}^{\sigma}$ is the Ricci tensor, $R_{\alpha \beta \gamma}^{\sigma}$ is the Riemann tensor, and $G_{\alpha \beta}=R_{\alpha \beta}-\frac{1}{2} R g_{\alpha \beta}$ is the Einstein tensor.
} 


\section{$2.2 \quad$ Matter}

Now let us consider what happens when matter is coupled to the metric. The field equations then take the form

$$
G^{\alpha \beta}=8 \pi T^{\alpha \beta}
$$

(in units with $c=G=1$ ), where $T^{\alpha \beta}$ is the matter stress tensor. If the stress tensor involves no higher than first derivatives and the matter field equations are of second order in derivatives, then the equations $G^{0 \beta}=8 \pi T^{0 \beta}$ are again initial value constraint equations. However, if the matter action involves the covariant derivative, such as for example in a term like $\left(\nabla_{\alpha} u_{\beta}\right)\left(\nabla^{\alpha} u^{\beta}\right)$ (where $u^{\beta}$ is a vector field), then variation of the metric will produce (among other things) terms involving the second derivatives of the metric and matter fields, so $T^{\alpha \beta}$ will generally involve second time derivatives. Then $G^{0 \beta}=$ $8 \pi T^{0 \beta}$ will not be constraint equations.

The difference from the vacuum case might seem puzzling since, when the matter satisfies its equation of motion, its stress tensor is divergence-free, $\nabla_{\alpha} T^{\alpha \beta}=0$. Together with the Bianchi identity this yields the equation $\nabla_{a}\left(G^{\alpha \beta}-8 \pi T^{\alpha \beta}\right)=0$, and it is tempting to argue by analogy with the Bianchi identity case that validity of this equation implies that $G^{0 \beta}-8 \pi T^{0 \beta}$ can have no second time derivatives. This would be incorrect however, since the equation uses the equations of motion of the matter field, so does not hold identically for all matter fields. In order to discover the true constraint equations in theories like this, what we need to do is find the analog of the Bianchi identity, that is, the identity that follows from the diffeomorphism invariance of the action.

\section{Any metric theory with tensor matter}

Consider then a diffeomorphism invariant action $S[g, \psi]$ that is a functional of the metric $g_{\alpha \beta}$ and some tensor matter field of any rank, $\psi_{\alpha \ldots \ldots}^{\beta \ldots}$. For notational simplicity I will assume $\psi$ is a $(1,1)$ tensor in what follows but it should be obvious how to modify the equations to allow for the general case. A diffeomorphism induces the variation (6) for the metric and

$$
\delta_{\xi} \psi_{\alpha}^{\beta}=\mathcal{L}_{\xi} \psi_{\beta}^{\alpha}=\xi^{\gamma} \nabla_{\gamma} \psi_{\beta}^{\alpha}+\psi_{\gamma}^{\beta} \nabla_{\alpha} \xi^{\gamma}-\psi_{\alpha}^{\gamma} \nabla_{\gamma} \xi^{\beta}
$$

for the tensor. Actually the Lie derivative is independent of the metric, so all the Christoffel symbols in (9) cancel out, but to maintain manifest covariance and to avoid the need to explicitly write the $\sqrt{-g}$ factor it is more convenient to use covariant derivatives. 
The variation of the action under a diffeomorphism is

$$
\delta_{\xi} S=\int E^{\alpha \beta} \delta_{\xi} g_{\alpha \beta}+H_{\beta}^{\alpha} \delta_{\xi} \psi_{\alpha}^{\beta},
$$

where

$$
E^{\alpha \beta}=\frac{\delta S}{\delta g_{\alpha \beta}}, \quad H_{\beta}^{\alpha}=\frac{\delta S}{\delta \psi_{\alpha}^{\beta}} .
$$

$E^{\alpha \beta}=0$ is the metric field equation, and $H_{\beta}^{\alpha}=0$ is the matter field equation. Now we insert the diffeo variations (6) and (9) into (10), integrate by parts on all derivatives of $\xi^{\alpha}$, and redefine some dummy indices to obtain

$$
\delta S=\int\left[\nabla_{\alpha}\left(-2 E_{\beta}^{\alpha}-H_{\gamma}^{\alpha} \psi_{\beta}^{\gamma}+H_{\beta}^{\gamma} \psi_{\gamma}^{\alpha}\right)-H_{\gamma}^{\alpha} \nabla_{\beta} \psi_{\alpha}^{\gamma}\right] \xi^{\beta} .
$$

Since the action is diffeomorphism invariant, this variation must vanish for all vector fields $\xi^{\beta}$, so we infer an identity,

$$
\nabla_{\alpha}\left(-2 E_{\beta}^{\alpha}-H_{\gamma}^{\alpha} \psi_{\beta}^{\gamma}+H_{\beta}^{\gamma} \psi_{\gamma}^{\alpha}\right)=H_{\gamma}^{\alpha} \nabla_{\beta} \psi_{\alpha}^{\gamma}
$$

This generalizes the Bianchi identity (21). Note that, unlike the latter, it is not just the statement that a certain tensor is divergence-free, but rather that the divergence is equal to some other quantity. It includes the matter fields and holds off-shell, i.e. for all field configurations.

Now to identify the initial value constraint equations, we expand the covariant divergence on the left hand side of (13) in terms of partial derivatives and Christoffel symbols as in (3). The term $\partial_{0}\left(-2 E_{\beta}^{0}-H_{\gamma}^{0} \psi_{\beta}^{\gamma}+H_{\beta}^{\gamma} \psi_{\gamma}^{0}\right)$ will have one higher time derivative order than any other term in (13). Since (13) holds identically for all fields, we infer that the quantity

$$
-2 E_{\beta}^{0}-H_{\gamma}^{0} \psi_{\beta}^{\gamma}+H_{\beta}^{\gamma} \psi_{\gamma}^{0}
$$

has time derivative order that is one less than the highest order in the field equations $E^{\alpha \beta}=0$ and $H_{\beta}^{\alpha}=0$. Also, it is a combination of components of the field equations, so it vanishes when the field equations hold. We thus arrive at the conclusion that the initial value constraints are given by

$$
\mathcal{C}_{\beta} \equiv-2 E_{\beta}^{0}-H_{\gamma}^{0} \psi_{\beta}^{\gamma}+H_{\beta}^{\gamma} \psi_{\gamma}^{0}=0 .
$$

For a general rank tensor matter field $\psi_{\ldots}^{\cdots}$ there would be one term in $\mathcal{C}_{\beta}$ for each index. Each covariant matter index contributes $-H_{\cdots 0}^{\cdots 0} \psi_{\cdots \beta}^{\cdots}$, and each contravariant index contributes $H_{\cdots \beta}^{\cdots} \ldots \psi_{\cdots} \cdots \cdots$. For multiple matter fields, there would be similar terms for each matter field. The generalized Bianchi identity (13) implies that the constraints are automatically preserved in time if they hold at one time and the matter field equations and other components of the metric field equations hold at all times. 


\section{Examples}

We now apply this result to three examples. The first example is EinsteinMaxwell theory. The matter field is a covariant vector potential $A_{\alpha}$, and the matter Lagrangian is $-\frac{1}{4} F^{\alpha \beta} F_{\alpha \beta}$, with $F_{\alpha \beta}=\partial_{\alpha} A_{\beta}-\partial_{\beta} A_{\alpha}$. The Maxwell stress tensor has no second derivative terms, so the quantities $E^{0 \beta}=-G^{0 \beta}+$ $8 \pi T^{0 \beta}$ involve no second time derivatives. Nevertheless, it is also true that $C_{\beta}$ in (15) has no second time derivatives. The extra term in $C_{\beta}$ in this case is $-H^{0} A_{\beta}$, so it must be that also $H^{0}$ has no second time derivatives. Indeed, $H^{0}=\nabla_{\alpha} F^{\alpha 0}$, and the antisymmetry of $F^{\alpha \beta}$ implies that the $\alpha$ index must be spatial. This field equation corresponds to $\nabla \cdot \mathbf{E}=0$, the initial value constraint for Maxwell's equations.

For the case of Einstein-aether theory $[8$ the tensor matter is the aether vector $u^{\alpha}$, and the Lagrangian contains Christoffel symbols, for instance in the term $\left(\nabla_{\alpha} u_{\beta}\right)\left(\nabla^{\alpha} u^{\beta}\right)$, so the stress tensor has second time derivatives. The constraint equations are

$$
\mathcal{C}_{\beta}^{\text {æther }} \equiv-2 E_{\beta}^{0}+u^{0} H_{\beta}=0
$$

where $H_{\beta}=0$ is the aether field equation, which contains second time derivatives that cancel those in $E_{\beta}^{0}$ when the two are added with the coefficients prescribed in (16). A special feature of this theory is that the field equation has no component in the direction of $u^{\beta}$, i.e. $H_{\beta} u^{\beta}=0$. That is because $u^{\beta}$ is constrained to be a unit timelike vector, hence all of its variations are orthogonal to it, $u_{\beta} \delta u^{\beta}=03$ Thus the $0-u$ component of the Einstein equation, $E_{\beta}^{0} u^{\beta}=0$, is an initial value constraint without any additional terms involving the aether field equation. In particular, on a hypersurface to which the aether is orthogonal, the $u u$ component of the aether stress tensor has no second derivatives in the $u$ direction.

The final example is Hořava gravity [9, 10]. I will first describe it in the original $3+1$ formulation with a preferred time, and then explain how the constraints work in its fully covariant formulation. In the original form, this theory has a fixed time function, defined up to reparameterizations, that determines a foliation of spacetime. The remaining symmetry is the time dependent spatial diffeomorphisms on the constant time surfaces. The field variables are the spatial metric, the shift vector, and the lapse scalar. I will discuss only the version of this theory in which the lapse $N$ is allowed to depend on the spatial coordinates, and in which the action contains all terms up to two derivatives, including[11] a term proportional to $\left(\partial_{i} N\right)\left(\partial^{i} N\right) / N^{2}$

\footnotetext{
${ }^{3}$ If the unit constraint is imposed by variation of a Lagrange multiplier, this statement holds only after the multiplier is solved for and inserted back into $H_{\beta}$.
} 
(which is invariant under time dependent rescaling of $N$ since $\partial_{i}$ denotes the spatial gradient). This theory has three first class constraints that generate spatial diffeomorphisms in the Hamiltonian formulation, and a fourth constraint that is second class, because the theory lacks surface deformation symmetry except for global time reparameterization [12, 13, 14, 4] This fourth constraint is the field equation for the lapse, and is an elliptic equation in spatial derivatives, so determines the lapse in terms of the other variables.

In the covariant formulation 11, 15, the field variables are the spacetime metric and a scalar field $T$, with a symmetry under reparameterizations of $T \rightarrow f(T)$. The two derivative action is just that of Einstein-aether theory, with aether vector $u_{\alpha}=N \partial_{\alpha} T$ and $N=\left(g^{\alpha \beta} \partial_{\alpha} T \partial_{\beta} T\right)^{-1 / 2}$. Because the "matter" field $T$ is a scalar, the generalized Bianchi identity (13) takes the form

$$
\nabla_{\alpha}\left(-2 E_{\beta}^{\alpha}\right)=H \nabla_{\beta} T
$$

where $H=-\nabla_{\alpha}\left[N\left(\delta_{\beta}^{\alpha}-u^{\alpha} u_{\beta}\right) \delta S / \delta u_{\beta}\right]$ is the $T$ field equation[15]. The covariant derivative of $u^{\alpha}$ appears in the action so, although the matter is a scalar field, the aether part of $E_{\beta}^{\alpha}$ has derivatives of order two on $g_{\alpha \beta}$ and three on $T$, while $H$ has derivatives of one higher order on each field variable. It is not until the gauge $x^{0}=T$ is chosen that the time derivative order of the theory drops to two: the field variable $T$ disappears from the equations, and the derivative orders of $E_{\beta}^{\alpha}$ and $H$ remain two and three respectively, but the third derivative in the $H$ field equation is always in a spatial direction. Since both field equations are now second order in time derivatives, the gaugefixed identity (17) implies that all four quantities $E_{\beta}^{0}$ have no second time derivatives. (The right hand side of (17) has no component orthogonal to $u^{\alpha}$ anyway, so even if $H$ had third time derivatives, one could have still inferred that the time-space components $E_{i}^{0}$ have no second time derivatives.) That is, the constraints are of the usual form, with no need to add a term involving the matter field equation.

\section{Discussion}

The constraint equations are essential input for calculation of evolution from initial data. That evolution could be in a timelike direction, as in ordinary time evolution, or a spacelike direction, as e.g. in the case of finding static or stationary solutions to field equations. In particular, the constraint equations for Einstein-aether theory on a timelike surface of constant radial coordinate were used in [16] to find static, spherically symmetric black

\footnotetext{
${ }^{4}$ On a compact spatial manifold there is one global first class surface deformation constraint that generates time reparameterizations [13].
} 
hole solutions to that theory. The constraints on a spacelike hypersurface in spherical symmetry were used in [4] to study perturbative stability of solutions in various modified gravity theories including Einstein-aether theory. The constraints for a spherically symmetric, spacelike hypersurface to which the aether is orthogonal were worked out for Einstein-aether theory in [17, and used in a numerical simulation of collapse of matter to form a black hole. An interesting application of the generalization beyond spherical symmetry would be to numerical simulation of axisymmetric black hole collapse, which could yield at late times a stationary rotating black hole solution.

\section{Acknowledgments}

I thank E. Barausse and T. Sotiriou for helpful discussions, and D. Garfinkle for useful comments on a draft of this article. This work was supported in part by the National Science Foundation under grants PHY-0601800 and PHY-0903572.

\section{References}

[1] J. Stachel, "Einstein's search for general covariance, 1912 - 1915," in Einstein and the History of General Relativity, Eds. D. Howard \& J. Stachel (Birkhäuser, 1989), pp. 63-100.

[2] P. Bergmann, "Non-Linear Field Theories," Phys. Rev. 75 (1949) 680.

[3] M. D. Seifert, R. M. Wald, "A General variational principle for spherically symmetric perturbations in diffeomorphism covariant theories," Phys. Rev. D75, 084029 (2007). gr-qc/0612121.

[4] M. D. Seifert, "Stability of spherically symmetric solutions in modified theories of gravity," Phys. Rev. D76, 064002 (2007). gr-qc/0703060.

[5] Weinberg, S., Gravitation and Cosmology: Principles and Applications of the General Theory of Relativity, (John Wiley \& Sons, Inc., 1972).

[6] L. D. Landau and E. M. Lifshitz, The Classical Theory of Fields, 4th ed. (Butterworth-Heinemann, 1975).

[7] J. Stachel, "The Cauchy Problem in General Relativity-The Early Years," in Studies in the History of General Relativity, Eds. J. Eisenstaedt \& A. J. Kox (Birkhäuser, 1992), pp. 407-418. 
[8] T. Jacobson, "Einstein-aether gravity: A Status report," PoS QG-PH, 020 (2007) [arXiv:0801.1547 [gr-qc]].

[9] P. Hořava, "Quantum Gravity at a Lifshitz Point," Phys. Rev. D79, 084008 (2009). arXiv:0901.3775 [hep-th]].

[10] T. P. Sotiriou, "Hořava-Lifshitz gravity: a status report," J. Phys. Conf. Ser. 283, 012034 (2011). arXiv:1010.3218 [hep-th]].

[11] D. Blas, O. Pujolas, S. Sibiryakov, "Consistent extension of Hořava gravity," Phys. Rev. Lett. 104, 181302 (2010).

[12] J. Kluson, "Note About Hamiltonian Formalism of Healthy Extended Hořava-Lifshitz Gravity," JHEP 1007, 038 (2010). arXiv:1004.3428 [hep-th]].

[13] W. Donnelly, T. Jacobson, "Hamiltonian structure of Hořava gravity," arXiv:1106.2131 [hep-th]].

[14] J. Bellorin and A. Restuccia, "Consistency of the Hamiltonian formulation of the lowest-order effective action of the complete Hořava theory," arXiv:1106.5766 [hep-th].

[15] T. Jacobson, "Extended Hořava gravity and Einstein-aether theory," Phys. Rev. D81, 101502 (2010). arXiv:1001.4823 [hep-th]].

[16] E. Barausse, T. Jacobson and T. P. Sotiriou, "Black holes in Einsteinaether and Hořava-Lifshitz gravity," Phys. Rev. D 83, 124043 (2011) arXiv:1104.2889 [gr-qc]].

[17] D. Garfinkle, C. Eling and T. Jacobson, "Numerical simulations of gravitational collapse in Einstein-aether theory," Phys. Rev. D 76, 024003 (2007) arXiv:gr-qc/0703093. 